\title{
Understanding Energy Behaviors of Thread Management Constructs
}

\author{
Gustavo Pinto \\ Federal University of Pernambuco \\ ghlp@cin.ufpe.br
}

\author{
Fernando Castor \\ Federal University of Pernambuco \\ castor@cin.ufpe.br
}

\author{
Yu David Liu \\ SUNY Binghamton \\ davidL@binghamton.edu
}

\begin{abstract}
Java programmers are faced with numerous choices in managing concurrent execution on multicore platforms. These choices often have different trade-offs (e.g., performance, scalability, and correctness guarantees). This paper analyzes an additional dimension, energy consumption. It presents an empirical study aiming to illuminate the relationship between the choices and settings of thread management constructs and energy consumption. We consider three important thread management constructs in concurrent programming: explicit thread creation, fixed-size thread pooling, and work stealing. We further shed light on the energy/performance trade-off of three "tuning knobs" of these constructs: the number of threads, the task division strategy, and the characteristics of processed data. Through an extensive experimental space exploration over real-world Java programs, we produce a list of findings about the energy behaviors of concurrent programs, which are not always obvious. The study serves as a first step toward improving energy efficiency of concurrent programs on parallel architectures.
\end{abstract}

Categories and Subject Descriptors D.1.3 [Concurrent Programming]: Parallel programming; C.4 [Performance of Systems]: Design Studies

General Terms Performance and Measurement

Keywords Energy Consumption, Performance, Thread Management, Multi-threaded Programming, Java

\section{Introduction}

IT energy consumption keeps rising steeply in spite of advances in many areas [2], and energy-efficient solutions are

Permission to make digital or hard copies of all or part of this work for personal or classroom use is granted without fee provided that copies are not made or distributed for profit or commercial advantage and that copies bear this notice and the full citation on the first page. Copyrights for components of this work owned by others than the author(s) must be honored. Abstracting with credit is permitted. To copy otherwise, or republish, to post on servers or to redistribute to lists, requires prior specific permission and/or a fee. Request permissions from permissions@ acm.org.

OOPSLA '14, October 20-24, 2014, Portland, OR, USA.

Copyright is held by the owner/author(s). Publication rights licensed to ACM. ACM 978-1-4503-2585-1/14/10...\$15.00.

http://dx.doi.org/10.1145/2660193.2660235 highly sought after across the compute stack. Among them, those on hardware/architecture [14-16, 19, 35, 37], operating systems [10, 25, 32, 41], and runtime systems [7, 33, 40] are more established. A number of solutions from higher levels of the compute stack - such as program analysis [4, 13], programming models [3, 6, 18, 34, 36], and applications [31, 42] - are also proposed in recent years. A higher-level study is often endowed with a broader application space. For example, programming model solutions can bring energy-aware programmers into energy optimization. Despite their promise, few language-level or applicationlevel energy-efficient solutions address concurrent software running on parallel architectures [4, 9, 33, 39]. This is unfortunate for at least two reasons: (1) thanks to the proliferation of multicore CPUs, concurrent programming is a standard practice in modern software engineering [38]; (2) a CPU with more cores (say 32) often consumes more power than one with fewer cores (say 1 or 2). Energy optimization over programs on such platforms has the potential to yield larger savings, but may also face more challenges [15, 16].

We believe a first step to optimize energy consumption of concurrent programs is to gain a comprehensive understanding of their energy behaviors. This paper presents an empirical study to illuminate and understand energy behaviors of Java concurrent programs on multicore architectures. In particular, our study is unique in its focus on how programmer decisions - the choices and settings of thread management constructs - may impact energy consumption and its close relative, performance. Our research is motivated by the following questions:

- RQ1. Do alternative thread management constructs have different impacts on energy consumption?

- RQ2. What is the relationship between the number of threads and energy consumption?

- RQ3. What is the relationship between task division strategies and energy consumption?

- RQ4. What is the relationship between data volume/access and energy consumption? 
To answer RQ1, we select three thread management constructs influential in concurrent language design:

- Explicit threading ("the Thread style"): programmers manually map logically independent units of work to threads, i.e., the scheduling unit of the virtual machine and/or the underlying operating system. Explicit threading is the most widely used approach in Java multithreaded programming [38].

- Thread pooling ("the Executor style"): programmers create a pool of threads - often fixed in size - and further submit logically independent units of work to the thread pool. The relationship between threads and the units of work is often 1:n. Threads select and execute submitted units of work from a centralized buffer managed by the language runtime. In Java, this mechanism is known as executors and is part of the java.util. concurrent library.

- Work stealing ("the ForkJoin style"): similar to thread pooling, programmers also create a pool of threads and submit logically independent units of work to the pool. What is unique to work stealing is that each thread maintains its own buffer of units of work. When one such buffer becomes empty, its maintaining thread may "steal" work from other threads. Its incarnation in Java is the ForkJoin framework [22].

Given these constructs, our investigation is further aimed at understanding how their settings _ - "tuning knobs" of concurrent programming for programmers - may impact energy consumption. Among them, the number of threads and the size of data are two classic knobs, addressing the dual control vs. data aspects of concurrency. Their respective impacts on energy consumption are focuses of our study. Tasks, i.e., logically independent units of work, have an intimate relationship with both. Just as the Executor and ForkJoin styles indicate, the ratio between the number of tasks and the number of threads is a design consideration of concurrent programmers. When the number of tasks increases while the size of data remains the same, each task will process a smaller "slice" of data, de facto tuning task granularity. We call the programmer job of dividing work to achieve desirable task granularity task division. The impact of task division strategies on energy consumption is another focus of our study.

Our study produces a list of findings, many of which are not obvious. We summarize them in Section 5, at the end of each RQ's discussion. We now highlight two of them.

First, our study reveals the context-dependent nature of the energy behaviors of thread management constructs. Each thread management construct has its own " 15 minutes of fame." Despite the highly complex landscape, some patterns do seem to recur. For example, as the number of threads for running a concurrent program continues to increase, we observe its energy consumption often increases first, and then decreases later, a phenomenon we term the $\Lambda$ curve. The shape of the curve differs significantly from the one that describes performance (execution time).

Second, our experiments further demonstrate that "faster" is not a synonym with "greener" for concurrent programs, and performance as an indicator to estimate energy consumption is unreliable at best - incorrect in most cases - for multi-threaded Java programs. We observed that a (faster) multi-threaded program execution generally does not consume less energy. In fact, the opposite is often true: the sequential variants of the benchmarks (i.e., executing a multi-threaded program with one thread) often exhibit the lowest energy consumption. That being said, the (effective) use of multi-threading does have its benefit in promoting energy efficiency: except for some embarrassingly serial benchmarks, multi-threading often achieves the best trade-off between energy consumption and performance. For example, one benchmark achieved a speedup of $9.5 \mathrm{x}$ when running with 32 threads, while its energy consumption only grew $1.97 x$.

Throughout our exploration, a recurring theme is to illuminate the intricate relationship between energy consumption and performance. There exists a rich literature on this topic [4, 6, 15, 16, 33, 34]. We enrich existing work by offering a programming-level perspective.

This paper makes the following contributions:

1. It describes an empirical study - the first of its kind to the best of our knowledge - to correlate energy behaviors of concurrent programs with thread management constructs and their knobs.

2. It conducts an extensive experimental exploration that involves a combination of factors, ranging from thread management constructs, the number of threads, task division strategies, task granularity choices, data sizes, and data access characteristics. The exploration carves out a landscape that involves thousands of distinct points in the experiment space. In addition, the paper describes a preliminary study on the stability and portability of our results under different settings of heap size, garbage collection, just-in-time compilation, and platforms.

3. It offers insights into energy behaviors of real-world concurrent Java programs, with a detailed list of often nonobvious findings.

\section{Related Work}

Studying energy efficiency of concurrent programs at the application/language level is an emerging direction. Most of the existing work concentrates on energy behaviors in the presence of synchronization. Under this backdrop, our work is unique in its focus on the impact of programming models for managing thread execution and program design choices on energy consumption. Park et al. [28] developed several synchronization-aware runtime techniques to bal- 
ance the trade-off between energy and performance. Gautham et al. [9] studied the relative energy efficiency of synchronization implementation techniques (such as spin locks and transactions). A recent short paper [23] called for energy management based on different synchronization patterns, a concrete instance of which based on futures has been formally defined [24]. Trefethen and Thiyagalingam [39] surveyed energy-aware software, including multi-threaded programs with different workload settings. Bartenstein and Liu [4] designed a data-centric approach to improve energy efficiency for multi-threaded stream programs. Ribic and Liu [33] designed an algorithm to improve the energy efficiency of the work-stealing runtime of Intel Cilk Plus through managing the relative speed of threads. Pinto and Castor [29] recently conducted a preliminary study, paving the way for the extended study we present here.

There are many approaches for energy management of multi-threaded programs at the architecture- and OS-levels. Examples in the former category include investigating the impact of Dynamic Voltage and Frequency Scaling on multicore architectures [16], meeting power budget based on hardware performance counters [15], and leveraging hardware heterogeneity [19] and processor topology [35]. Examples in the latter include studying the impact of energy consumption based on workloads [10], thread schedules [25, 41], and thread migration [32]. Our work and related work cited here are complementary. Together, they attempt to understand energy behaviors of multi-threaded programs through the perspectives of different levels of the compute stack.

More broadly, there is a growing interest in understanding and managing energy consumption from software-centric approaches. Tiwari et al. [37] correlated energy consumption with CPU instructions. Vijaykrishnan et al. [40] and Farkas et al. [7] performed two early studies on the energy consumption of the JVM. More recently, Hao et al. [13] designed a dynamic analysis to estimate energy consumption of Android bytecode. They also observed that there is no strong correlation between performance and energy consumption. Within the programming language community, it is an active area of research to design energy-aware programming languages, with examples such as Eon [36], Green [3], EnerJ [34], Energy Types [6], and LAB [18]. None of these software-centric energy management approaches focuses on multi-threaded programs.

Performance analysis of multi-threaded Java programs has a long history, leading to a rich literature we cannot cite in full. In recent years, there are numerous results based on the DaCapo benchmark suite [5] for this purpose. Kalibera et al. [17] conducted a comprehensive study on the benchmarks in Dacapo itself.

Lea [22] described the work stealing algorithm implemented by Java's ForkJoin framework. Work stealing was popularized by the Cilk language [8], and there is a growing

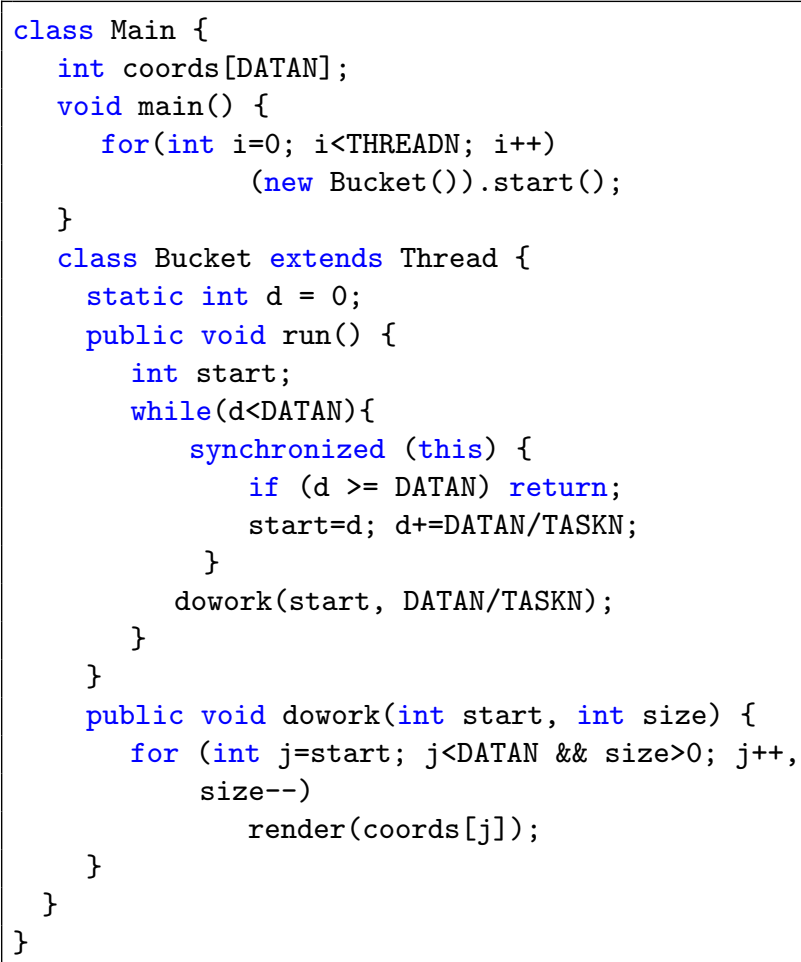

Figure 1. Concurrent Programming in Thread Style

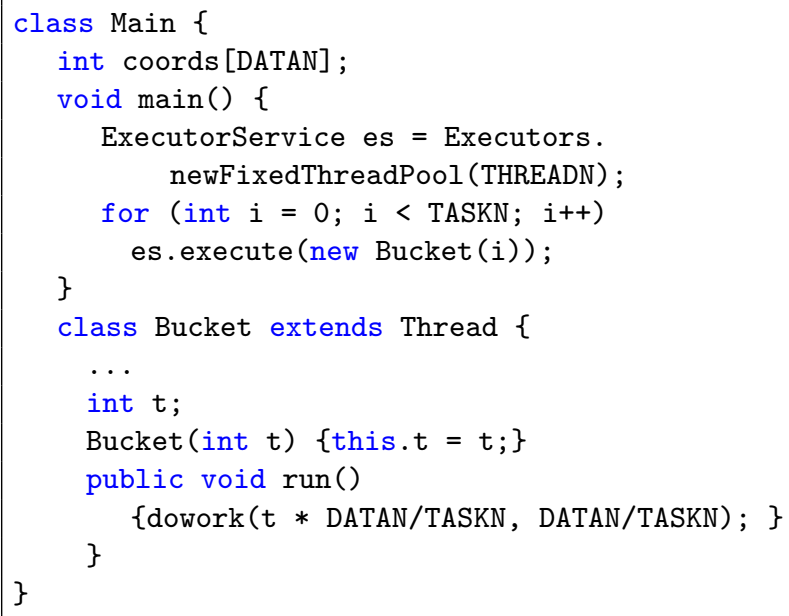

Figure 2. Concurrent Programming in Executor Style

interest in designing multi-threaded language runtimes with work-stealing thread management [12, 20, 33].

Earlier versions of Java use green threads [27]. The term is unrelated to energy consumption; it refers to VM-managed threads. After Java 1.3, green threads have been replaced by native threads, where programmer-created threads are directly mapped to OS threads. 


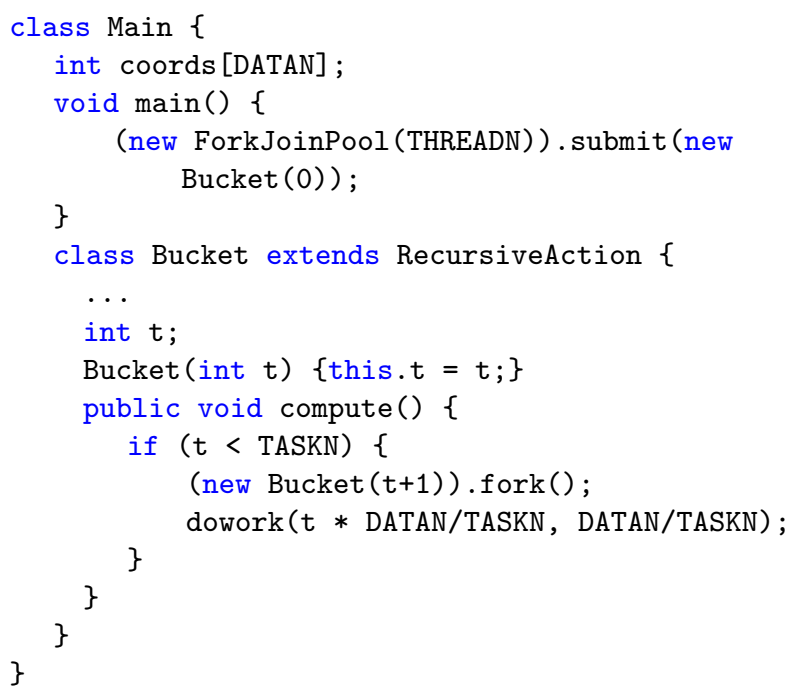

Figure 3. Concurrent Programming in Task-Centric ForkJoin Style

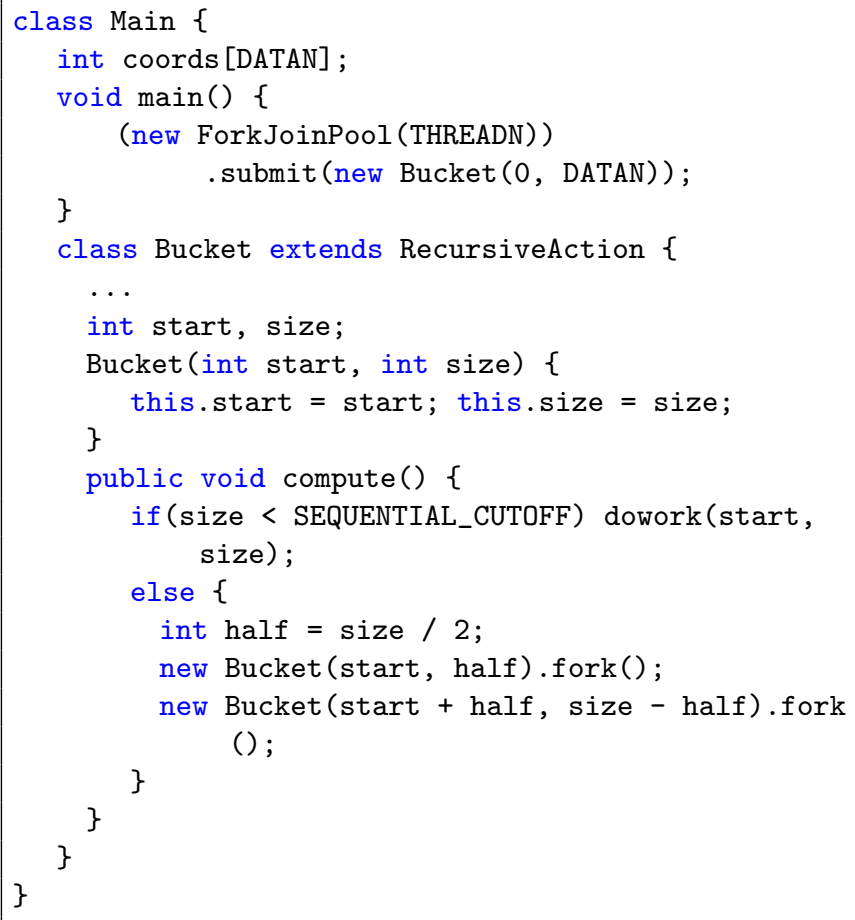

Figure 4. Concurrent Programming in Data-Centric ForkJoin Style

\section{Programming Patterns for Thread Management}

We use an (overly) simplified version of the sunflow benchmark [5] to illustrate the distinct programming patterns of the three thread management constructs. Figure 1 and Figure 2 demonstrate the Thread style and the Executor style, respectively. Figure 3 and Figure 4 both demonstrate the ForkJoin style, with a difference we will explain shortly. The

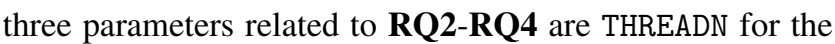
number of threads (RQ2), and TASKN for the number of tasks (RQ3), and DATAN for the data size (RQ4), respectively.

The sunflow benchmark centers around a rendering algorithm (ray tracing) where coordinates are stored in array coords and method render takes one coordinate to render. The rendering logic is encompassed in a method called dowork. The coordinates to be processed by a dowork invocation are a range of size number of consecutive elements beginning at index start. For brevity, the code snippets here omit the body of the render method, and further omit program logic unrelated to our discussion here, such as postrendering processing (typically performed through placing a barrier at the end of the main function).

In the Thread style, the program explicitly bootstraps THREADN threads, through messaging the start method of a Bucket object, whose class is a subclass of the JDK Thread class. The run method of the Bucket class (an inner class of Main in the example) is executed by each bootstrapped thread. Here, each thread continuously processes tasks through a busy while loop, and each task is defined as executing an instance of dowork. Since there are TASKN tasks, each task will work on a "slice" of coordinates of size DATAN/TASKN. A global counter $d$ is used to track the size of data that has been processed, and the counter is accessed from within a synchronized block.

In the Executor style, THREADN threads are created in a fixed-size thread pool, managed by an instance of the ExecutorService class of the JDK. The inner class Bucket now only encompasses a task and its run method only executes the dowork method (definition identical to that in Figure 1) once. Each task is identified by a counter $t$. In the main method, TASKN tasks will be managed by the pool of THREADN threads. The submission for management is achieved through the use of the execute method of the ExecutorService object.

The ForkJoin style is similar to the Executor style in that a fix-sized pool - the ForkJoinPool object - will manage THREADN threads. Unlike Executor however, ForkJoin adopts a work stealing algorithm to manage threads. Instead of submitting all tasks to a centralized service such as in Executor, each thread under a work-stealing scheduler maintains its own localized queue-like structure, called a deque, for tasks. A thread running out of tasks (a thief) will "steal" a task from the deque of another randomly selected thread (a victim). The runtime behavior of work stealing is defined through a classic yet sophisticated algorithm, with subtleties detailed in prior work [8, 22].

From a programming perspective, the thread pool for work stealing is initially only submit'ed with one task, an object subclassed from the JDK class RecursiveAction. A thread in the pool will pick up the task, i.e., run its compute method. The compute method may further fork new tasks "on the go," where forking can be viewed as placing the task 
on the thread's own deque. Such a task in turn may either be picked up by the current thread, or be stolen and picked up by other threads in the pool. Both Figure 3 and Figure 4 follow this common pattern.

Recursively dividing work into smaller tasks is a distinct programming pattern for programs written in work-stealing languages or language frameworks. As it turns out, different task division strategies exist, with Fig. 3 highlighting a task-centric task division strategy, and Fig. 4 demonstrating a data-centric task division strategy. In the task-centric approach, we directly fix the number of tasks (through TASKN), and keep a counter to track how many tasks have been forked so far. In contrast, the data-centric approach sets a sequential cutoff threshold to data, i.e., the size of data a task will work on, instead of explicitly setting and tracking the number of tasks. The two strategies lead to different programming patterns, and the choice is largely dependent on what is considered more natural to specific programs. It however should be pointed out that they are indeed two sides of the same coin for task granularity: given the overall data, fixing the number of tasks will implicitly set the data size per task, whereas fixing (sequential cutoff) data size will implicitly determine the number of tasks.

In the rest of the paper, we manually refactor each benchmark into the four programming patterns. Figures 1, 2, 3, and 4 serve as examples of what we view as "comparable" programs in our benchmarking process. We routinely fix two of the three parameters - THREADN, DATAN, TASKN (or its counterpart of sequential cutoff threshold) - and observe the impact on energy/performance when the 3rd parameter varies. For example, when THREADN and DATAN remain the same but TASKN increases, it is aligned with our intuition that tasks become more "fine-grained."

\section{Experiment Setup}

In this section we describe the benchmarks that we analyzed, the infrastructure and the methodology that we used to perform the experiments.

\subsection{Benchmarks}

We use a variety of benchmarks for evaluation, listed as follows. Benchmarks 1-3 are from a Debian-based language benchmark suite ${ }^{1}$. Benchmark 4 was developed by us. Benchmark 5 is a modification of a program originally developed for a work-stealing language system [20]. The rest of the benchmarks are from the well-known DaCapo suite [5].

1. knucleotide: This benchmark takes a DNA sequence, and counts the occurrences and their frequencies of nucleotide patterns. The memory-intensive benchmark employs string manipulation intensively. There is no syn-

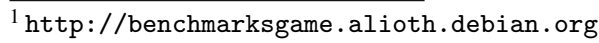

chronization point in the program, but one atomic variable is used.

2. mandelbrot: A mandelbrot is a mathematical set of points whose boundary is a distinctive and easily recognizable two-dimensional fractal shape. Mandelbrot set images are created by sampling complex numbers and determining for each one whether the result tends toward infinity when a particular mathematical operation is iterated on it. According to its website, this benchmark spends $99 \%$ of the time using CPU, and uses I/O only to print the results. There is no synchronization point in the program, but one atomic variable is used.

3. spectralnorm: The spectral norm is the maximum singular value of a matrix. The benchmark is CPU-intensive, and scales up well in multicore machines. This benchmark synchronizes threads using a barrier, and uses one atomic variable.

4. largestimage: This benchmark performs a recursive search into the file system, looking for image files. During traversal, it keeps track of the number of image files it encountered and the largest among them. This benchmark has two synchronization points and is strongly I/Obound.

5. n-queens: This benchmark is the classic $\mathrm{N}$-queens chessboard game, placing $\mathrm{N}$ chess queens on an $\mathrm{NxN}$ chessboard so that no two queens attack each other. It is a computationally intensive, CPU-bound problem. This benchmark does not have synchronization points, but uses one atomic variable.

6. sunflow: renders a set of images using ray tracing ${ }^{2}$.

7. xalan: transforms XML documents into HTML.

8. h2: executes a number of transactions against a model of a banking application, in a style similar to JDBCbench.

9. tomcat: runs a set of queries against a Tomcat server retrieving and verifying the resulting webpages.

We selected the benchmarks based on their diverse characteristics. For instance, according to a recent study [17], sunflow scales well when the number of CPU cores increases, h2 scales rather poorly, and xalan is the middleof-the-road benchmark in terms of scalability. Benchmark largestimage is I/O-intensive, knucleotide is memoryintensive, and benchmarks mandelbrot, $\mathrm{n}$-queens, and spectralnorm are CPU-intensive.

For the benchmarks, DATAN represents the number of patterns for knucleotide, the size of the vector for both mandelbrot and spectralnorm, the size of a matrix for $\mathrm{n}$-queens, the number of directories for largestimage, the size of the image for sunflow, the number of converted

\footnotetext{
${ }^{2}$ The description for the DaCapo benchmarks was taken directly from the DaCapo website: http://www.dacapobench.org/
} 
files for xalan, and the number of database transactions for h2.

\subsection{Experimental Environment}

Unless noted otherwise, all experiments were conducted on a machine with $2 \times 16$-core AMD Opteron 6378 processors (Piledriver microarchitecture) running Debian 3.2.46-1 x8664 Linux (kernel 3.2.0-4-amd64) and 64GB of DDR3 1600 memory and Oracle HotSpot 64-Bit server VM, JDK version 1.7.0_11, build 21. All experiments were performed with no other load on the OS. We conform to the default settings of both the OS and the JVM. Several default settings are relevant to this context: (1) the power management of Linux is the default ondemand governor, which dynamically adjusts CPU core frequencies based on system workloads. (2) For the JVM, the parallel garbage collector is used, and just-intime (JIT) compilation is enabled. The initial heap size and maximum heap size are set to be $1 \mathrm{~GB}$ and $16 \mathrm{~GB}$ respectively. We run each benchmark 10 times within the same JVM; this is implemented by a top-level 10-iteration loop over each benchmark. The reported data is the average of the last 3 runs. We justify this decision in Section 6 .

Energy consumption is measured through current meters over power supply lines to the CPU module. Data is converted through an NI DAQ and collected by NI LabVIEW SignalExpress with 100 samples per second and the unit of the current sample is deca-ampere (10 ampere). Since the supply voltage is stable at $12 \mathrm{~V}$, energy consumption is computed as the sum of current samples multiplied by $12 \times 0.01 \times 10$. We measured the "base" power consumption of the OS when there is no JVM (or other applications) running. The reported results are the measured results modulo the "base" energy consumption.

\section{Study Results}

In this section, we report the results of our experiments. Results for RQ1 and RQ2 are presented in Section 5.1, which describes the impact of different thread management constructs in the presence of varying numbers of threads. In Section 5.2 we attempt to answer RQ3 by investigating the impact of different task division strategies. Finally, in Section 5.3 we present answers to RQ4 by exploring different data characteristics.

\subsection{Energy Behaviors with Alternative Programming Abstractions and Varying Numbers of Threads}

In this group of experiments, we fix the number of tasks and the size of the data, and study how variations on the number of threads and the choice of different thread management constructs impact energy consumption. The results of our experiments are presented in Figure 5. Here, the odd rows are energy consumption results, whereas the even rows are the corresponding performance results.
The $\Lambda$ Curve. One interesting observation throughout our study is that energy consumption typically increases as the number of threads increases, and then gradually decreases as the number of threads approaches the number of CPU cores. In the energy consumption figures, the curves typically display a $\Lambda$ shape, which is why we term it $\Lambda$ curve. Nearly all benchmarks display the $\Lambda$ curve.

We believe the $\Lambda$ curve results from a combination of multicore processor characteristics and program performance traits. Under the default setting of the ondemand governor, power management modules of multicore CPUs work in an "adaptive" fashion: when a particular core stays idle, the operating frequency of the core will be dynamically adjusted to a lower level. When a 32-core CPU is only loaded with 4 threads for instance, a large number of cores will operate on the lowest frequency (the specific number of cores is likely to be slightly more than 4 , because of the execution of VM/OS threads). It is standard knowledge that power consumption is reduced when the operating frequency is lower. For that reason, a program running 4 threads is likely to consume less power than one running 8 threads. This helps us explain the / part of the $\Lambda$ curve.

To see why energy consumption often decreases after the initial increase, note that energy consumption, by definition, is the multiplication of power and time. As more threads are used, program execution time tends to shorten. The extent of the drop - the \ part of the $\Lambda$ curve - is determined by the increase in performance (and thus decrease in time) and the increase in power consumption. The greater the ratio between speedup and increase in power, the steeper the $\backslash$ part of the curve will be.

The specific shape details of the $\Lambda$ curve, including the "peaking" point and the slope of the increase/decrease, are application-specific. Take sunflow and h2 (in the Thread style) for example. Power consumption for the two benchmarks is $8.54 W$ and $6.87 W$ on average, respectively, when using 1 thread, and 88.05 $\mathrm{W}$ and $14.27 \mathrm{~W}$ when using 32 threads. Execution time is 17.74 and 9.92 seconds, respectively, when using 1 thread, and 1.34 and 10.38 seconds when using 32 threads. Since the power consumption for sunflow increases about 10x and performance improves $13 \mathrm{x}$, energy consumption in fact decreases. For h2 however - a benchmark known to scale rather poorly as the number of cores increases - power consumption increases $2.07 \mathrm{x}$ but execution time also increases $1.04 \mathrm{x}$, yielding $2.17 \mathrm{x}$ energy consumption. Thus, in this extreme case, the $\backslash$ part of the curve does not exist.

Embarrassingly Parallel vs. Embarrassingly Serial. Our selection of benchmarks range from "embarrassingly parallel" ones ( sunflow, tomcat, spectralnorm, and n-queens), to middle-of-the-road ones (xalan, knucleotide, and mandelbrot), to "embarrassingly serial" ones (h2, and to some extent largestimage)). The performance results of 


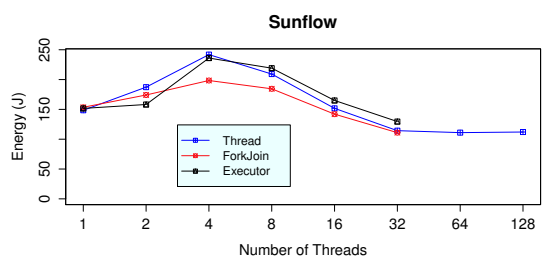

(a)

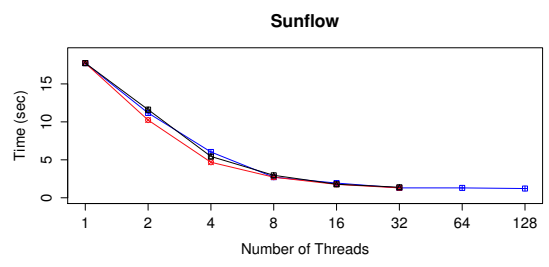

(d)

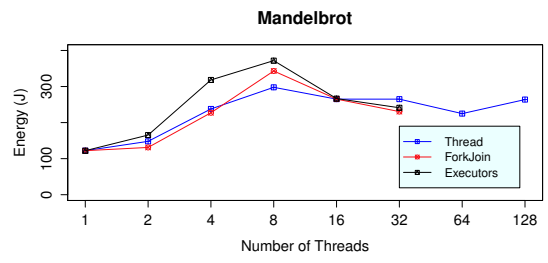

$(\mathrm{g})$

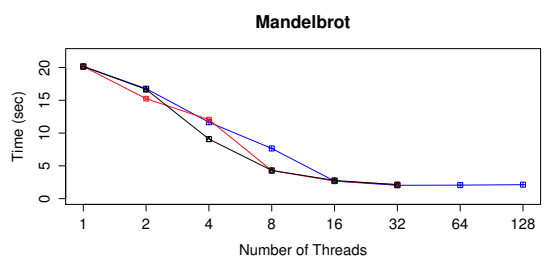

(j)

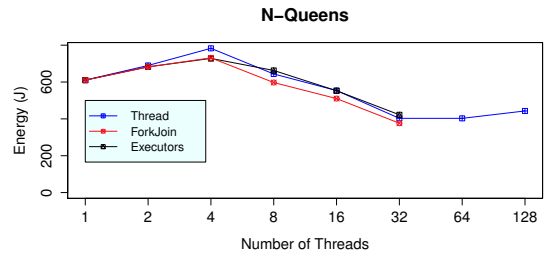

(m)

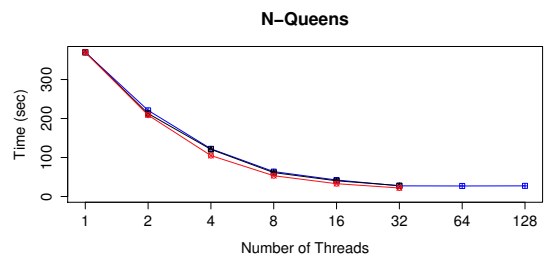

(p)

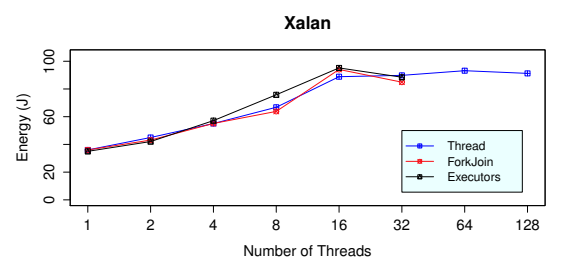

(b)

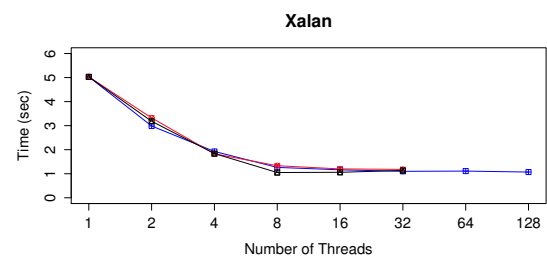

(e)

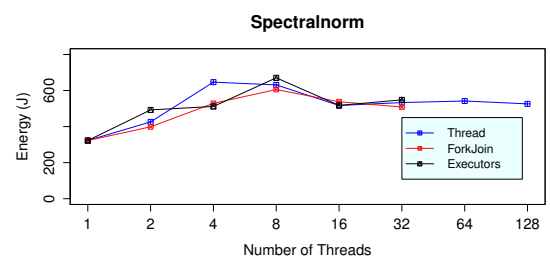

(h)

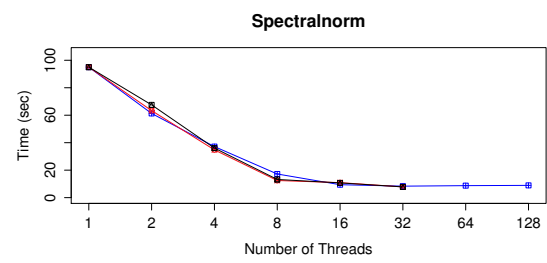

(k)

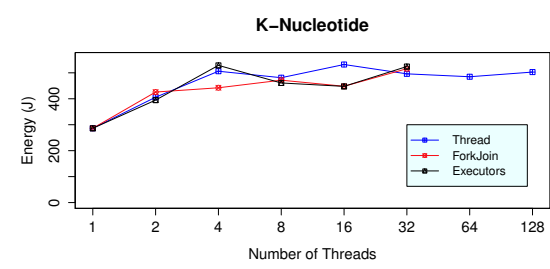

(n)

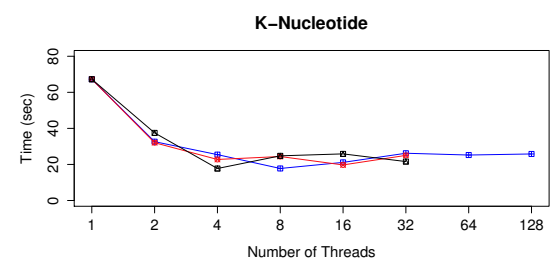

(q)

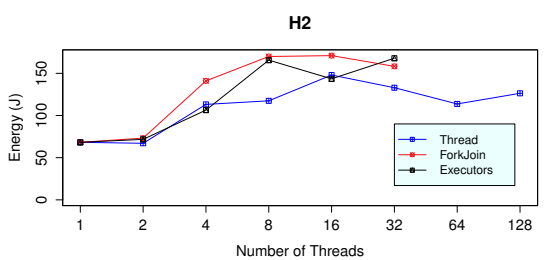

(c)

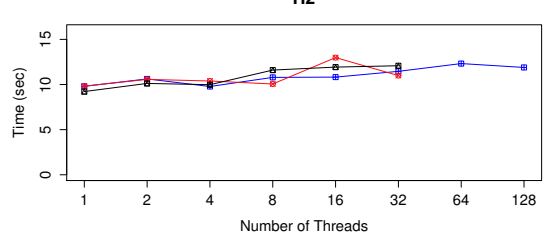

(f)

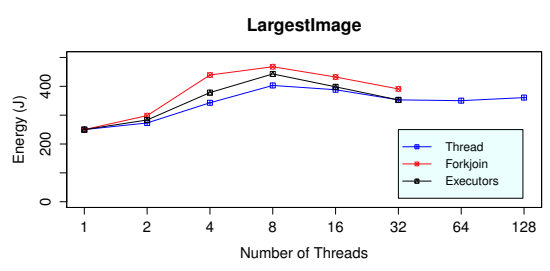

(i)

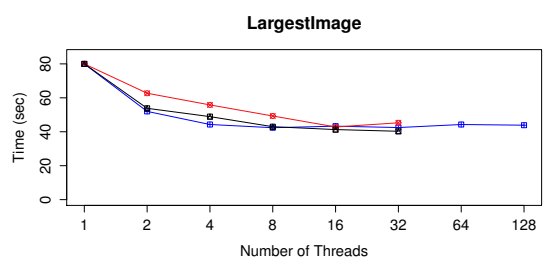

(1)

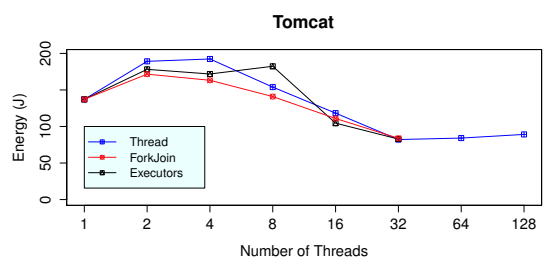

(o)

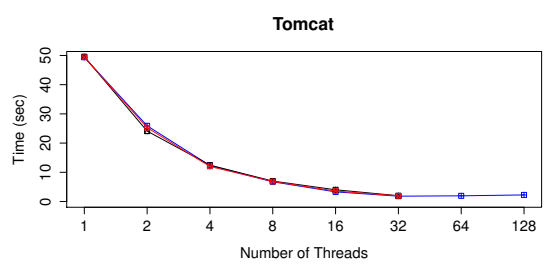

(r)

Figure 5. Energy/Performance with Alternative Programming Abstractions and Varying Numbers of Threads 
four Dacapo benchmarks - Figure 5(d)(e)(f)(r) — are consistent with recent studies (e.g., [17]).

We find the (more) embarrassingly parallel benchmarks are likely to "peak" earlier on the $\Lambda$ curve, i.e., reaching the highest energy consumption with the smallest number of threads. For example, sunflow's $\Lambda$ curve peaks at 4 threads, whereas xalan peaks at 16 . We think this is reasonable: the speed-up of sunflow is almost $8 \mathrm{x}$ when the number of threads increases from 1 to 8 (linear speedup), so the reduction in execution time can quickly offset the increase in power consumption early on. In comparison, xalan produces a $5 x$ speedup with the same variation in threads and its performance does not improve with more threads. Hence, its $\Lambda$ curve peaks later.

Faster $\neq$ Greener. $\quad$ In most of our benchmarks, additional threads would initially lead to improved performance; see Figure 5(d)(e) for example. Following the / part of the $\Lambda$ curve however, the energy consumption increases as the number of threads increases initially. Furthermore, for 6 of our 9 benchmarks, the lowest energy consumption was achieved by the sequential (1 thread) version. Being "faster" clearly has little correlation with being "greener" for concurrent programs on multicore architectures.

Moreover, since benchmarks "peak" at different parts of the $\Lambda$ curve, it is not possible to generalize that an improvement in time could be seen as an improvement in energy, and vice versa.

Which Programming Style Should I Use? As Figure 5 shows, it is possible to detect differences in the amount of energy used when different concurrent programming abstractions are employed. For some benchmarks, this difference is small, e.g., xalan in Figure 5(b)(e). However, the difference is more noticeable in others. Every programming abstraction may have its " 15 minutes of fame." In one configuration of sunflow, ForkJoin outperforms Thread and Executor by reducing energy consumption by $30 \%$, as shown in Figure 5(a). In one configuration of h2 however, ForkJoin underperforms Thread and Executor by increasing energy consumption by 50\%, as shown in in Figure 5(c). Our experiments do show that there are scenarios where one style is more likely to outperform the others, which we summarize now.

First, the Thread style performs well in I/O-bound (such as largestimage) benchmarks. One possible explanation is that in I/O-bound benchmarks, the instruction pipeline has a higher likelihood to stall. In such a scenario, the Thread style defers context switching and/or load balancing to the OS, which appears to be efficient. The Executor style and the ForkJoin style build an additional layer of thread management on top. Unfortunately, this higher layer of decision making may disagree with the OS, missing some opportunities for context switching in the presence of long-latency I/O operations.
Second, the energy consumption of ForkJoin-style programming is sensitive to the degree of parallelism latent in the benchmarks. It outperforms the other two strategies when the benchmarks are embarrassingly parallel (e.g., Figures $5(\mathrm{a})(\mathrm{h})(\mathrm{m})(\mathrm{o}))$, but underperforms the other two strategies in the presence of more serial benchmarks, such as h2. We believe this can be explained through the nature of the work stealing algorithm: it excels through balancing the deques of individual threads. For benchmarks involving significant serial portions, synchronization (such as a barrier) is often used during the execution of a task. The work stealing algorithm is oblivious to such intra-task synchronizations, preventing tasks from being stolen and thus suppressing load balancing. In other words, the impacts of stealing a task with long synchronization delays and one without are clearly different, and the natural strength of work stealing in balancing tasks among threads is broken when long intra-task synchronization delays are present. Along this line, the Executor style performs slightly better, but still not as efficiently as the Thread style. One possible reason is that an Executor needs to manage a queue of worker threads. Updates to the queue are protected from clients by a lock, thus increasing synchronization costs when a new task is submitted. Such overhead does not exist in the Thread style.

Energy-Performance Trade-offs. An energy-related question arises when we move from single-threaded programming to multi-threaded programming, or from 16 threads to 32 threads: what is the relationship between energy consumption and performance? One well-known metric to evaluate the energy/performance trade-off is the Energy-Delay Product (EDP): the product of energy consumption and execution time [21]. We compute the EDP for the benchmarks, with results presented in Fig. 6, where a smaller EDP value indicates the more favorable trade-off.

We observed that a parallel execution is generally more favorable for energy-performance trade-offs than its singlethreaded counterpart. This is particularly true for embarrassingly parallel programs: the EDP for sunflow with 32 threads is only $5.8 \%$ of its single-threaded execution. The degree of improvement on EDP appears to be in sync with the potential of parallelism in applications, and for specific benchmarks, increasing the number of threads is most likely not aligned with the improvement of EDP. For instance, when the number of threads increases for xalan from 8 to 16, EDP for all three programming constructs deteriorates, since there is very little performance improvement and an average growth of more than $30 \%$ in energy consumption. The most unfortunate case among our benchmarks is perhaps h2. As the number of threads increases, the benchmark produces no gain in performance, but its energy consumption triples. As a result, EDP degrades as we move from sequential to parallel execution.

Overpopulating Cores with Threads. For the Thread style of thread management, we have also constructed experi- 

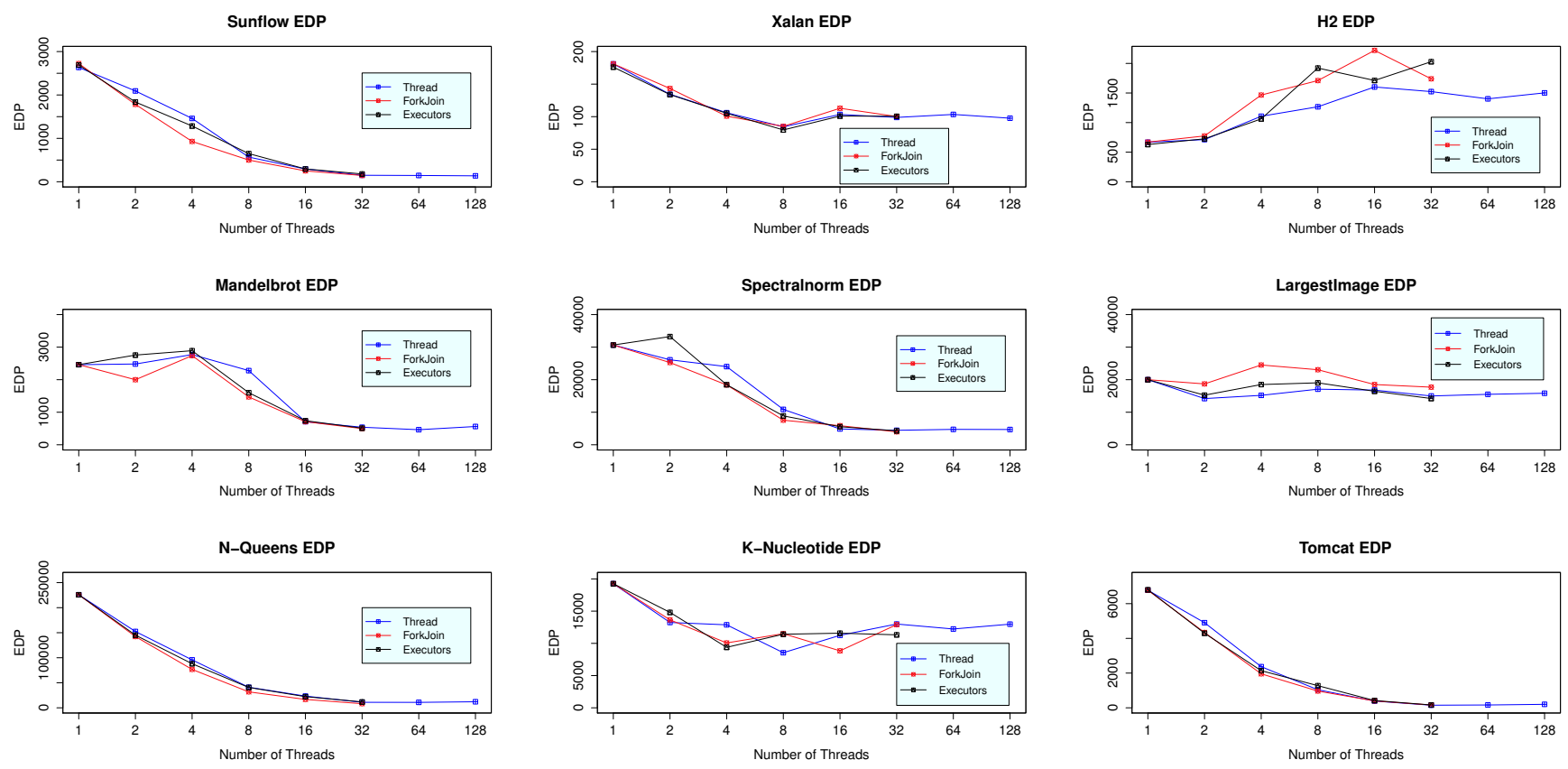

Figure 6. EDP (a smaller value is better)

ments where the number of threads goes beyond the number of cores. In all experiments, we did not notice significant change in energy consumption. This suggests that the JVM and the OS are well-versed in handling cases where threads outnumber cores. Make no mistake: the number of context switches does increase as the cores become more overpopulated with threads. For instance, in the sunflow benchmark, the number of context switches increases $3.57 \mathrm{x}$ when the number of threads varies from 32 to 128 threads, as Figure 7 shows.

We choose not to perform experiments over the cases where there are more threads than CPU cores for Executor and ForkJoin styles. The "comparable" (Section 3) implementation would create a thread pool that outnumbers the number of cores. We do not believe that is the intended use for these thread management constructs.

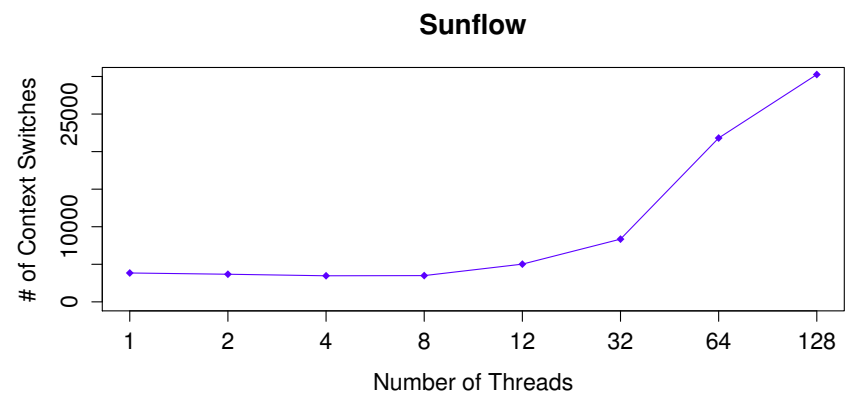

Figure 7. Context Switches and Thread Overpopulation
RQ1 Summary: Different thread management constructs have different impacts on energy consumption. For I/O-bound programs, the Thread style incurs the least energy consumption, whereas the ForkJoin style has the most. For embarrassingly parallel benchmarks, the opposite holds.

RQ2 Summary: The relationship between energy consumption and the number of threads often forms the $\Lambda$ curve. Being faster is not synonymous with being greener. Sequential execution often leads to the least energy consumption, whereas parallel execution leads to improved energy/performance trade-off for nonembarassingly-serial programs.

\subsection{Energy Behaviors and Task Division Strategies}

In this section, we fix the number of threads and the size of data, and study how the variations on the number of tasks have effects on energy consumption. To thoroughly explore the experimental space, we further refine our benchmarks into two versions: a task-centric division strategy and a datacentric division strategy as we first introduced in Section 3.

Task Granularity with Task-Centric Division. In this style, we divide the work based on TASKN. Figure 8 demonstrates the effect of task granularity on xalan benchmark ${ }^{3}$. We observed a similar energy consumption behavior in the

\footnotetext{
${ }^{3}$ Throughout the rest of the paper, we select a subset of benchmarking results to illustrate ideas. Additional graphs can be found in the technical report [30].
} 
other benchmarks. Here the data exhibit remarkable uniformity: the number of tasks submitted/executed as logically independent units of work has little impact on energy consumption, independently of the thread management construct.
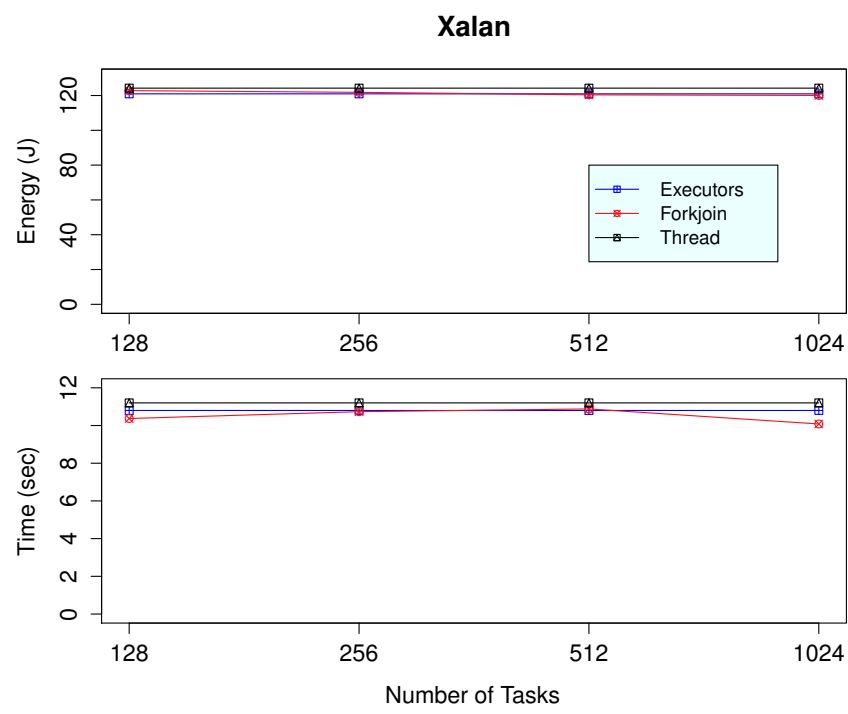

Figure 8. Energy/Performance and Task-Centric Division

At first glance, the results may be disappointingly "boring." We believe, however, that no news is news. The data reveal that task granularity matters little to energy consumption of concurrent programs. For instance, we have at a point increased the number of tasks to 1024 , for a benchmark whose overall DATAN is 2048. In other words, every task only takes 2 pieces of data. In this case, no noticeable energy consumption increase was observed. Its version in the Executor style submits 1024 tasks to the ExecutorService and its version in the ForkJoin style recursively creates 1024 RecursiveAction objects. Though such programming patterns may appear to be "extreme," our experiments show they place little burden on energy consumption.

Task Granularity with Data-Centric Division. Under a data-centric approach, ForkJoin can also be seen as a divideand-conquer algorithm, where in each recursive call new tasks are spawned until a certain threshold is reached. Using this approach, Figure 9 shows the energy/performance behavior of different sequential threshold configurations for the sunflow benchmark, where each recursive call spawns two tasks to divide work into halves. We choose not to perform experiments for the Thread and Executor styles because their programming patterns do not naturally fall into the divide-and-conquer style as ForkJoin does.

There are three observations from this set of experiments. First, energy consumption and execution time both increase when the sequential threshold changes from 135 to 405 , a $2.66 x$ increase in energy consumption and a $2.64 x$ increase
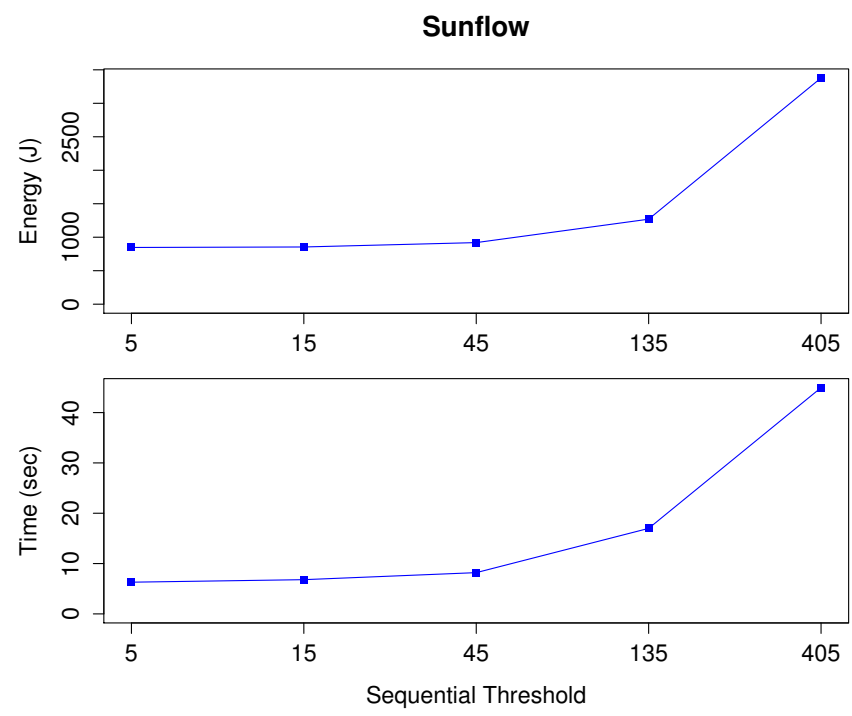

Figure 9. Energy/Performance and Data-Centric Division

in execution time. In this example, the benchmark operates over an array of 2048 positions. Thus, when we use 405 as the sequential cutoff threshold, the benchmark creates less than 10 tasks and operates on at most as many CPU cores. With the majority of the cores idle, the benchmark is not able to take advantage of the multiprocessors. As the sequential threshold reduces to 135 , the program operates on more cores. As the $\Lambda$ curve suggests, both the energy consumption and the execution time reduce for sunflow.

Second, the overhead of scheduling a high number of tasks does not seem to impact energy consumption. This phenomenon appears to recur in all benchmarks, and it is consistent with our findings for the task-centric experiments.

Third, energy consumption and execution time do not always increase in sync. For example, there is a small energy consumption variation $(7.85 \%)$ when the sequential threshold changes from 45 to 135 . Performance, on the other hand, degrades $23.8 \%$. One possible reason is that, when tasks become more coarse-grained, it is less likely that a ForkJoin thief will steal a task, because the total number of available tasks decreases. Thus, after few unsuccessful attempts, the processor goes idle and the average power consumption decreases.

Asymmetric Workload. So far, we have created tasks where the data is divided uniformly. Another important characteristic to take into consideration is the use of asymmetric workloads. With different amounts of work, some ForkJoin workers will finish their work faster than others. Hence, the likelihood of steals may increase. Figure 10 shows the average number of steals per task granularity in the presence of symmetric load, a random asymmetric work division, and an 80-20 asymmetric work division. 


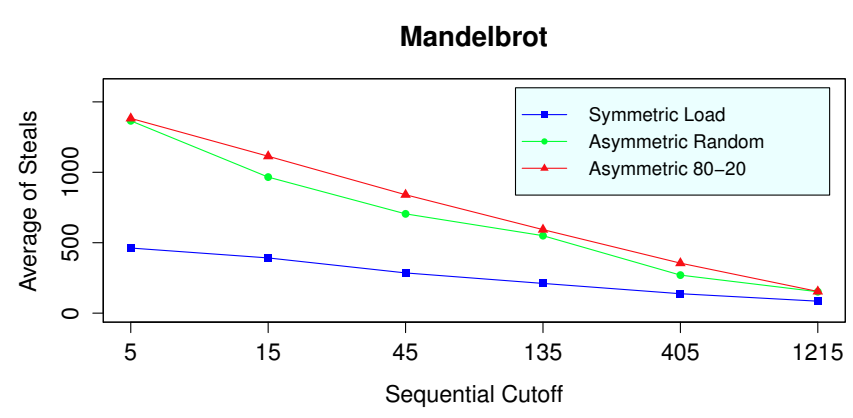

Figure 10. Number of Steals and Task Granularity

The figure shows that the number of steals is strongly correlated to the symmetric vs. asymmetric nature of task workloads. Further, the number of steals is also correlated to task granularity: the smaller the tasks, the greater the number of steals. We observed an average energy savings of $3.26 \%$ using asymmetric workloads. We have experienced similar results in CPU-bound benchmarks, as Figure 11 shows.

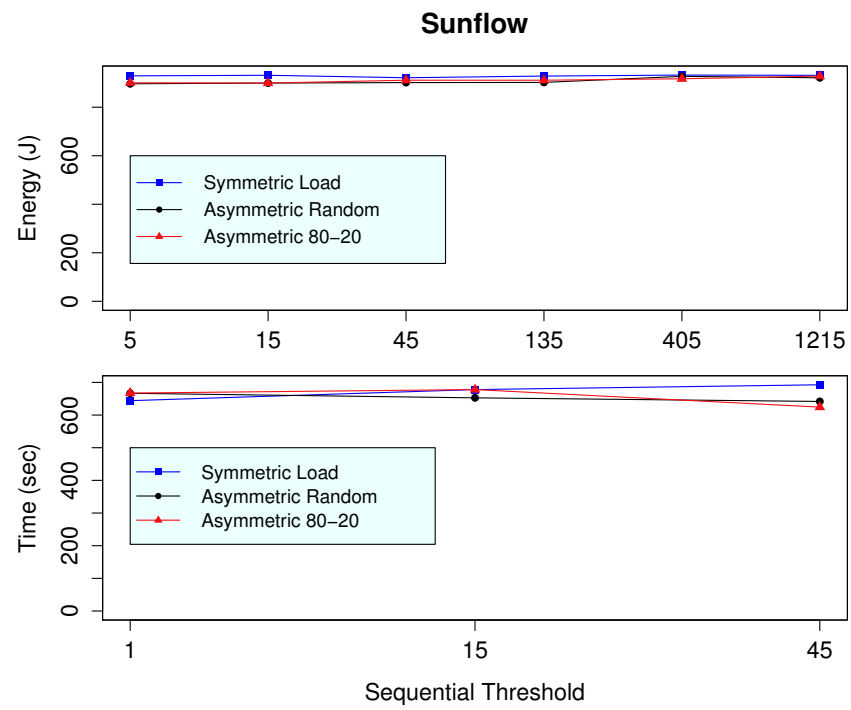

Figure 11. Energy/Performance with Asymmetric Workload

The Width of Forking. The ForkJoin-style can be configured to divide the work into $n$ desirable tasks, instead of two per recursive call, which we term the width of forking. We have analyzed 4 different forking widths. For the sunflow benchmark in Figure 12, we observed a negligible difference of energy consumption from 2 to 4 forks, and from 4 to 8 forks per recursive call (about $0.96 \%$ and $1.21 \%$ respectively). From 8 forks to 16 forks, however, we observed an increase of $5.78 \%$ over the total energy consumption, and a similar increase in the execution time of $5.67 \%$. This result is consistent with the other benchmarks. The experiment here suggests that excessive forking width can lead to increased energy consumption.

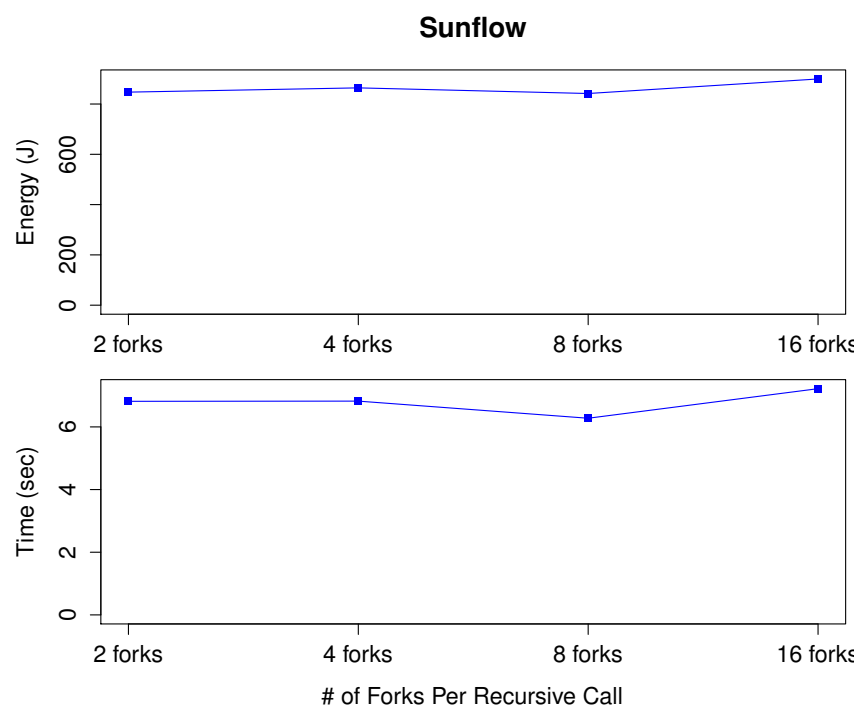

Figure 12. Energy/Performance and Forking Width

RQ3 Summary: for task-centric division, the granularity of tasks appears to matter little to energy consumption. For data-centric division, asymmetric workloads in ForkJoin are more energy-friendly, and excessive forking width can lead to increased energy consumption.

\subsection{Energy Behaviors and Data}

We now focus on RQ4, studying the impact of data - its size and access patterns - on program energy behaviors.

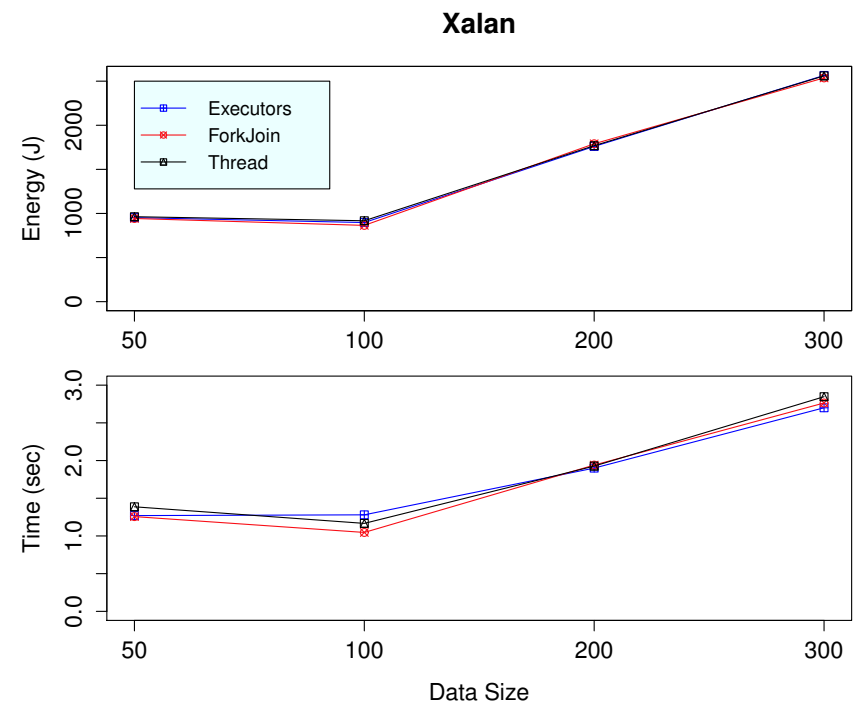

Figure 13. Energy/Performance and Data Size

Data Size. Fixing the number of threads and the number of tasks, we now study how the variations on data size have effect on energy consumption. Figure 13 shows the energy behavior for the xalan benchmark, where the analogous 
DATAN value (as used in examples Fig. 1/2/3/4) represents the number of XML files to be converted. THREADN and TASKN were fixed at 32 and 256, respectively.

As predicted, energy consumption increases when a larger number of files are processed. Observe however, the increase in energy consumption is not necessarily linear to data size. Generally speaking, the precise relationship is application-specific: it depends on the algorithm complexity relative to the data size. In cases of data-parallel benchmarks, one phenomenon we observe is that the curve is often convex, especially for the part of the curve where the data sizes are relatively small. Take xalan for instance. When data size increases from 50 to 100 , the energy consumption and performance remain almost unchanged. We think this has to do with the programming pattern itself. In dataparallel programs, there is usually a barrier at the end of data processing, and performance is determined by the slowest processing thread. When overall data size is small, the execution time of processing each data "slice" is also small. Variations on processors and scheduling may contribute to a larger proportion on the progress of individual threads, and differences in data size may be masked. When data size increases, the masking effect is reduced.

In xalan, the energy behaviors with the 3 thread management constructs are nearly identical, but there is a detectable difference in performance for the three constructs, with ForkJoin taking the least time and Executor taking the most. Since energy is the accumulated effect of power over time, this indicates ForkJoin is likely to have completed the task faster with a higher power consumption. Work stealing systems are most known for their ability for load balancing, where CPU core idling is reduced, improving performance while presenting fewer opportunities for cores to fall into lower power modes. This phenomenon is reduced when data size becomes larger, because data processing time would be proportionally larger, reducing the relative effect of frequent steals.

Data Sharing vs. Copying. We now study how memoryintensive tasks may impact energy consumption. Many ForkJoin benchmarks (in the style of data-centric division) operate on an indexable data structure, with subtasks operating on partitions of this data structure. During recursion, it is often necessary to split the data structure into smaller pieces on which the newly forked tasks can work. One possible solution is to copy part of the data structure and use it for the newly forked tasks. Given an array-based data structure, each recursive call in this scenario will create $n$ new arrays, where $n$ is the width of forking. However, an alternative solution is to share this array, with newly forked tasks operating on contiguous partitions of this data structure. In all the experiments we have reported so far, sharing is the default strategy for data use. In the next set of experiments, we modify each benchmark to so that the forked tasks operate on copies of the data structure, instead of working on
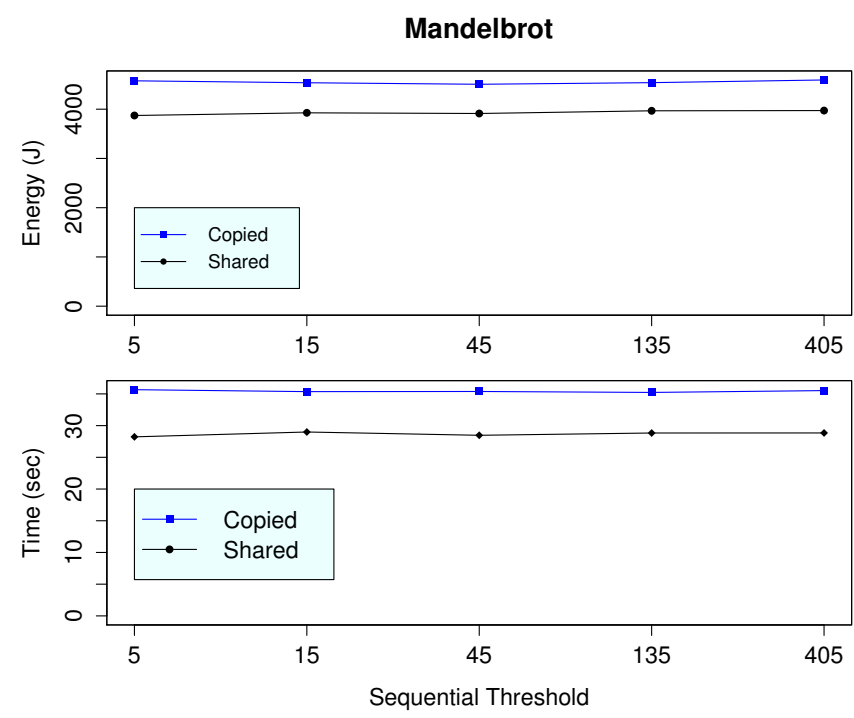

Figure 14. Energy/Performance and Data Sharing Strategies

in-place data. In Figure 14 we compare the two approaches using the mandelbrot benchmark.

As the figure shows, we experienced an energy consumption increase of $15.38 \%$ when copying is used. In the meantime, performance degrades by $20.85 \%$. In other words, copying has severe impact on both energy and performance. In cases where the newly forked tasks are unlikely to lead to data races, this set of experiments demonstrate that a ForkJoin programmer should use shared data structures as much as possible. Furthermore, observe that copying has a more severe impact on performance than energy. This is indeed natural: when a long-latency main memory request is issued, the issuing cores can often be reduced to a lower frequency, and a lower level of power consumption. (Recall again that energy is the multiplication of power and execution time.)

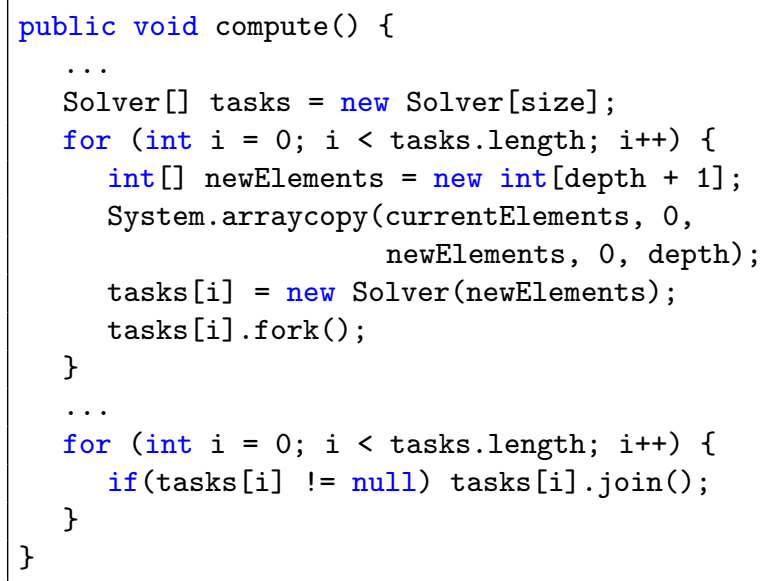

Figure 15. ForkJoin: Spreading Out Data Copying 


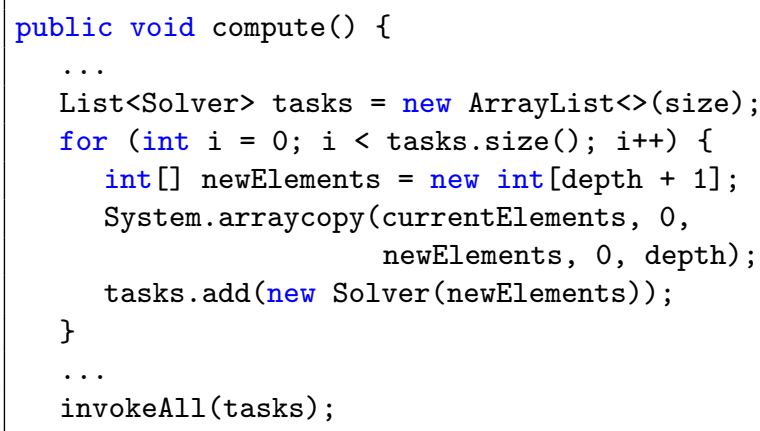

Figure 16. ForkJoin: Aggregating Data Copying

Data Locality. Next, we investigate the impact of data locality on energy consumption. We modify the (data copying flavor of the) $n$-queens benchmark into two versions: Figure 15 and Figure 16. The two versions are functionally identical. In the first version, the execution of a task follows the sequence of ababababc where a is copying memory for a subtask, $\mathrm{b}$ is forking the subtask, and $\mathrm{c}$ is computing the current task. In the second version, the execution of a task follows the sequence of aaaacbbbb ${ }^{4}$.

Which version should fare better? On the surface, the second version indeed admits less parallelism on the execution of the current task: it forks the subtasks only after the current task has finished. Therefore, it cannot be executed in parallel with any of the a steps or the c step. Our benchmarking results on the other hand show the opposite: the second version yields energy savings of $10.11 \%$ and a performance improvement of $10.66 \%$.

We hypothesize that data locality plays an important role. Note that in the first version we interspersed data copying with thread forking (together with other operations in a loop iteration). Any of the latter operations may potentially pollute the cache, increasing the chance of memory round-trips. In the second version however, the same memory area is repeatedly requested, leading to better data locality.

To further strengthen our belief that data locality is the main cause here, we also investigated the same two-version approach, but using a data sharing strategy. There is no noticeable difference in energy consumption and performance for the two versions.

\footnotetext{
${ }^{4}$ The invokeall method in the second version is part of the Java ForkJoin API. It forks all tasks and then joins them all. Through inspecting its source code, we find no "magic" that would otherwise skew the results.
}

RQ4 Summary: Data size has non-linear impact on energy consumption. In data-parallel programs, the curve to demonstrate the relationship between data size ( $X$ axis) and energy consumption (Y-axis) is often convex. Performance is closely related to energy consumption in the presence of data size variations, but the two do not follow identical trajectories. Significant data copying leads to increased energy consumption, but its relative effect is often smaller than the performance loss it imposes. Data locality plays an important role in the energy consumption of multi-threaded programs.

\section{Threats to Validity}

In experimental systems research, a fundamental challenge is the vast number of factors across the compute stack. For instance, it is a valid question to ask whether different OS scheduling policies $[25,41]$, different processor and interconnect layouts [19, 35], and different VLSI circuit designs [1], have impact on results. They clearly all do. Our study takes a route common in experimental programming language research, by constructing experiments over representative system software and hardware, and the results are empirical by nature.

To take a step further, we seek to gain a preliminary understanding of how variations in the underlying system impact our results. In particular, we focus on configuration variations of the language runtime. The primary goal is to understand the stability and portability of our results.

Heap Size. Heap size settings are known to impact JVM performance (e.g., [11]). Figure 17 shows the energy consumption and performance under different settings of maximum heap sizes (to trigger GC) for sunflow; the rest of the JVM settings are identical to those described in Section 4. When maximum heap size is restricted to a very low level - such as 20MB for sunflow - both energy consumption and performance go higher significantly. We speculate the additional overheads result from VM allocation and garbage collection. Variations in energy consumption that stem from heap size appear to be small if the maximum heap size is higher. While examining this benchmark without setting a fixed maximum heap size, we observed that its heap usage reaches a peak of more than $50 \mathrm{MB}$ before $\mathrm{GC}$ is triggered. Fixing the heap size at $20 \mathrm{MB}$ may have triggered significantly more GC.
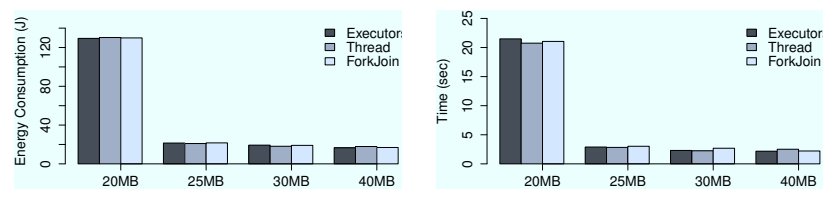

Figure 17. Heap Size Effect (sunflow, 32 threads, 256 tasks, 256 as image data size) 
Garbage Collection Strategy. To gain a preliminary understanding of how GC strategies may pose a threat to the validity of our results, we constructed experiments over 5 GC options over Hotspot: (a) SerialGC: the stop-the-world serial collector, (b) ParallelGC: the parallel collector, (c) ParallelOldGC: the parallel collector with data compression, (d) ConcMarkSweepGC: concurrent mark sweep collector, and (e) G1GC: the garbage-first collector. All have been specified by Oracle [26]. Figure 18 shows the results for xalan.
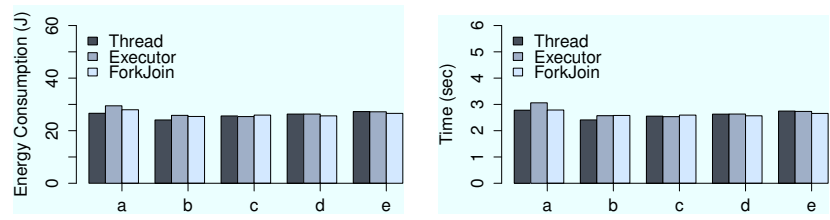

Figure 18. GC Effect (xalan, 32 threads, 64 tasks, 300 transformation files. GC strategies are: a: SerialGC, b: ParallelGC, c: ParalleloldGC, d: ConcMarkSweepGC, e: G1GC)

As shown, GC strategies do have observable impact on program energy consumption. In the context of this study, the effect is relatively mild, within $\pm 10 \%$. A precise relationship between GC and energy consumption is a complex topic beyond the scope of this paper.

Just-In-Time Compilation. Just-In-Time (JIT) compilation dynamically optimizes the program and is known to have significant impact on performance. Predictably, JIT also has direct impact on energy consumption. Figure 19 shows the effect of JIT on sunflow.

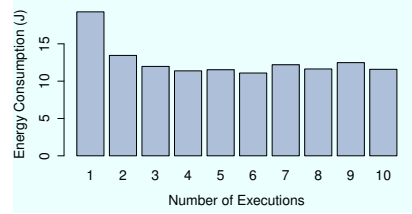

(a) Energy with JIT

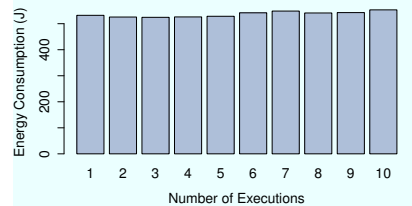

(c) Energy without JIT

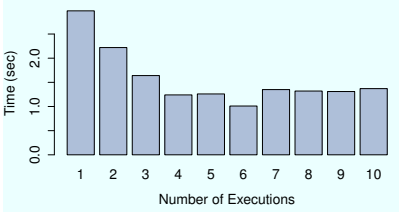

(b) Time with JIT

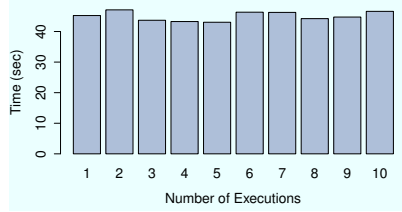

(d) Time without JIT
Figure 19. JIT Effect (sunflow, 32 threads, 256 tasks, 256 as image data size, 10 runs on $\mathrm{X}$-axis)

Here, the X-axis represents 10 "hot" runs of sunflow, i.e., a top-level loop that encompasses 10 executions within one JVM. With JIT, early runs incur higher energy/time overhead than later runs, as illustrated in Figure 19(a) and Figure 19(b). Also note that energy/performance behaviors do stabilize after a number of runs. With JIT disabled, both energy consumption and performance are uniform, as shown in Figure 19(c) and Figure 19(d). Both of them however are also significantly worse than their JIT counterparts.

Moreover, the growth of energy and time is not proportional. Execution time increases by $33 \mathrm{x}$ from using JIT to not using JIT, whereas energy consumption increases more than $45 x$. For instance, for the 10th sunflow execution, the average power (not energy) consumption using JIT was $85.47 \mathrm{~W}$, and when not using it was $118.35 \mathrm{~W}$. After a more detailed inspection of the data, we observed that although the JIT executions recorded the highest power consumption $(175.3 \mathrm{~W}$ using JIT and $166.3 \mathrm{~W}$ not using JIT), non-JIT dominates the executions with higher power consumptions (3rd quartile: $163.2 \mathrm{~W}$ ), that is, consuming more power, than the approach using JIT (3rd quartile: $154.6 \mathrm{~W}$ ). This result, combined with the enormous difference in performance between JIT and non-JIT yields the results from Figures 19(a) and 19(c).

In Section 4, we explained our data collection strategy as averaging the last runs of JIT-enabled executions. This decision stems from our observations here: (1) JIT-disabled executions incur energy/performance overhead unrealistic to common use of Java applications, and (2) later runs of JITenabled executions do stablize in terms of energy consumption and performance.

Platform Variations. As a final experiment, we ran some of the benchmarks on a different machine: an 8-core AMD FX-8150 processor (Bulldozer architecture) with 16GB of DDR 1600 memory, running Debian 3.2.46-1 Linux (kernel 3.2.0-4-amd64) and Oracle HotSpot 64-Bit server VM, JDK version 1.7.0_45, build 18. Figure 20 shows the results for n-queens benchmark.
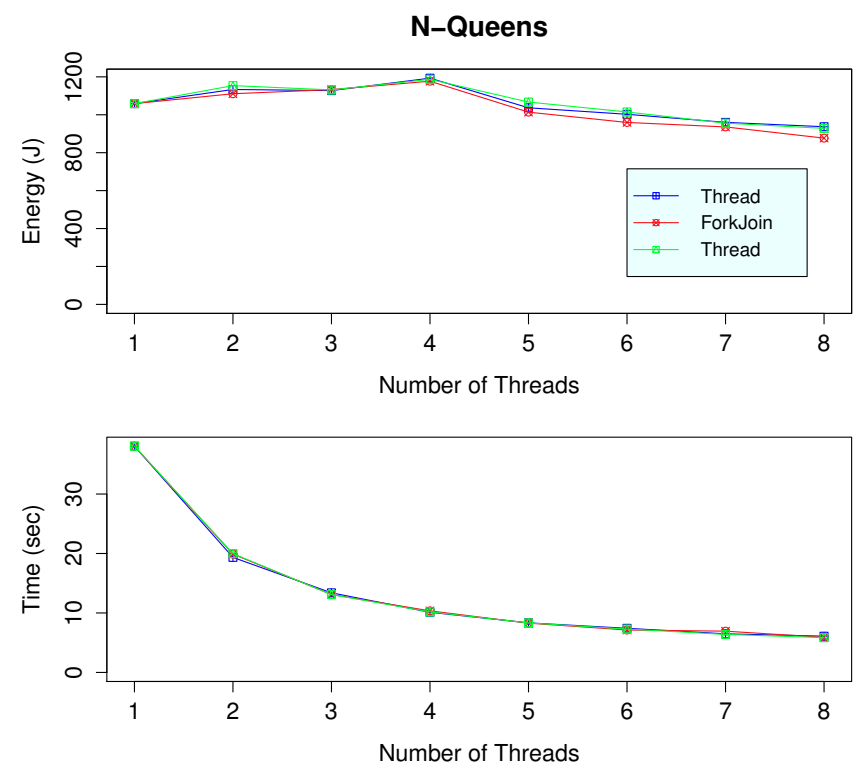

Figure 20. Energy/Performance on Alternative Platform 
The benchmarking results show similar trends. For instance, the $\Lambda$ curve recurs, peaking at 4 threads - the same behavior for this benchmark when using 32 processors. The thread management styles behave similarly when compared to the 32-core machine: ForkJoin still outperforms Thread and Executor.

\section{Conclusion}

In this paper, we present a study on how concurrent programming practices may have impact on energy consumption. Our results suggest that different constructs for managing concurrent execution can impact energy consumption in different ways, and energy consumption is determined by the choice of thread management constructs, the number of threads, the granularity of tasks, the size of the data, and the nature of data access. This study is a step toward a better understanding of the interplay between energy efficiency and performance.

In the future we intend to study the energy behaviors of different data structures and their various implementations within the Java language. We also plan to develop tools to support developers in systematically alternating between different parallel programming constructs while taking into account their energy consumption characteristics.

\section{Acknowledgments}

We would like to thank the anonymous reviewers for their helpful comments, and Irineu Moura for the valuable reviews. Gustavo is supported by CAPES/Brazil, Fernando is supported by CNPq/Brazil (306619/2011- 3, 487549/2012-0 and 477139/2013-2), FACEPE/Brazil (APQ-1367-1.03/12) and INES (CNPq 573964/ 2008-4 and FACEPE APQ-1037$1.03 / 08)$, and David is supported by a Google award and by US NSF (CCF-1054515). Any opinions expressed here are from the authors and do not necessarily reflect the views of the sponsors.

\section{References}

[1] A.Chandrakasan, S. Sheng, and R. Brodersen. Low power cmos digital design. IEEE Journal of Solid State Circuits, 27:473-484, 1992.

[2] J. Asafu-Adjaye. The relationship between energy consumption, energy prices and economic growth: time series evidence from asian developing countries. Energy Economics, 22(6):615 - 625, 2000.

[3] W. Baek and T. Chilimbi. Green: a framework for supporting energy-conscious programming using controlled approximation. In PLDI, 2010.

[4] T. Bartenstein and Y. Liu. Green streams for data-intensive software. In ICSE, 2013.

[5] S. Blackburn, R. Garner, C. Hoffmann, A. Khang, K. McKinley, R. Bentzur, A. Diwan, D. Feinberg, D. Frampton, S. Guyer, M. Hirzel, A. Hosking, M. Jump, H. Lee, J. Moss, A. Phansalkar, D. Stefanović, T. VanDrunen, D. von Dinck- lage, and B. Wiedermann. The dacapo benchmarks: java benchmarking development and analysis. In OOPSLA, 2006.

[6] M. Cohen, H. Steve Zhu, S. Emgin, and Y. Liu. Energy types. In OOPSLA, 2012.

[7] K. Farkas, J. Flinn, G. Back, D. Grunwald, and J. Anderson. Quantifying the energy consumption of a pocket computer and a java virtual machine. In SIGMETRICS, 2000.

[8] M. Frigo, C. Leiserson, and K. Randall. The implementation of the cilk-5 multithreaded language. In PLDI, 1998.

[9] A. Gautham, K. Korgaonkar, P. Slpsk, S. Balachandran, and K. Veezhinathan. The implications of shared data synchronization techniques on multi-core energy efficiency. In HotPower, 2012.

[10] R. Ge, X. Feng, W. Feng, and K.W. Cameron. Cpu miser: A performance-directed, run-time system for power-aware clusters. In ICPP, 2007.

[11] A. Georges, D. Buytaert, and L. Eeckhout. Statistically rigorous java performance evaluation. In OOPSLA, 2007.

[12] Y. Guo, J. Zhao, V. Cavé, and V. Sarkar. Slaw: A scalable locality-aware adaptive work-stealing scheduler. In IPDPS, 2010.

[13] S. Hao, D. Li, W. Halfond, and R. Govindan. Estimating mobile application energy consumption using program analysis. In ICSE, 2013.

[14] M. Horowitz, T. Indermaur, and R. Gonzalez. Low-power digital design. In Low Power Electronics, 1994. IEEE Symposium, 1994.

[15] C. Isci, A. Buyuktosunoglu, C. Cher, P. Bose, and M. Martonosi. An analysis of efficient multi-core global power management policies: Maximizing performance for a given power budget. In MICRO, 2006.

[16] A. Iyer and D. Marculescu. Power efficiency of voltage scaling in multiple clock, multiple voltage cores. In ICCAD, 2002.

[17] T. Kalibera, M. Mole, R. Jones, and J. Vitek. A black-box approach to understanding concurrency in dacapo. In OOPSLA, 2012.

[18] A. Kansal, T. Saponas, A. Brush, K. McKinley, T. Mytkowicz, and R. Ziola. The latency, accuracy, and battery (lab) abstraction: programmer productivity and energy efficiency for continuous mobile context sensing. In OOPSLA, 2013.

[19] R. Kumar, K. Farkas, N. Jouppi, P. Ranganathan, and D. Tullsen. Single-isa heterogeneous multi-core architectures: The potential for processor power reduction. In MICRO 36, 2003.

[20] V. Kumar, D. Frampton, S. Blackburn, D. Grove, and O. Tardieu. Work-stealing without the baggage. In OOPSLA, 2012.

[21] JamesH. Laros III, Kevin Pedretti, SuzanneM. Kelly, Wei Shu, Kurt Ferreira, John Vandyke, and Courtenay Vaughan. Energy delay product. In Energy-Efficient High Performance Computing, SpringerBriefs in Computer Science, pages 5155. Springer London, 2013.

[22] Doug Lea. A java fork/join framework. In Java Grande, 2000. 
[23] Y. Liu. Energy-efficient synchronization through program patterns. In GREENS, 2012.

[24] Y. Liu. Variant-frequency semantics for green futures. In PLACES'12, 2012.

[25] A. Merkel and F. Bellosa. Balancing power consumption in multiprocessor systems. In EuroSys, 2006.

[26] Oracle. Java hotspot garbage collection. http: //www.oracle.com/technetwork/java/javase/tech/ index-jsp-140228.html, 2014. [Online; accessed 21-Mar-2014].

[27] Oracle. Java language and virtual machine specifications. http://docs.oracle.com/javase/specs/, 2014. [Online; accessed 21-Mar-2014].

[28] S. Park, W. Jiang, Y. Zhou, and S. Adve. Managing energyperformance tradeoffs for multithreaded applications on multiprocessor architectures. In SIGMETRICS, 2007.

[29] G. Pinto and F. Castor. On the implications of language constructs for concurrent execution in the energy efficiency of multicore applications. In SPLASH, 2013.

[30] G. Pinto, F. Castor, and Y. Liu. Understanding energy behaviors of thread management constructs - remaining data. http://bit.ly/oopsla-techreport. Accessed: 201408-22.

[31] G. Pinto, F. Castor, and Y. Liu. Mining questions about software energy consumption. In MSR, 2014.

[32] K. Rangan, G. Wei, and D. Brooks. Thread motion: finegrained power management for multi-core systems. In ISCA, 2009.

[33] H. Ribic and Y. Liu. Energy-efficient work-stealing language runtimes. In ASPLOS, 2014.
[34] A. Sampson, W. Dietl, E. Fortuna, D. Gnanapragasam, L. Ceze, and D. Grossman. Enerj: Approximate data types for safe and general low-power computation. In PLDI, 2011.

[35] A. Solernou, J. Thiyagalingam, M. Duta, and AnneE. T. The effect of topology-aware process and thread placement on performance and energy. In Supercomputing, Lecture Notes in Computer Science, 2013.

[36] Jacob Sorber, Alexander Kostadinov, Matthew Garber, Matthew Brennan, Mark D. Corner, and Emery D. Berger. Eon: a language and runtime system for perpetual systems. In SenSys'07, pages 161-174, 2007.

[37] V. Tiwari, S. Malik, and A. Wolfe. Power analysis of embedded software: A first step towards software power minimization. IEEE Transactions on VLSI Systems, 2:437-445, 1994.

[38] W. Torres, G. Pinto, B. Fernandes, J. Oliveira, F. Ximenes, and F. Castor. Are java programmers transitioning to multicore?: a large scale study of java floss. In TMC, 2011.

[39] A. Trefethen and J. Thiyagalingam. Energy-aware software: Challenges, opportunities and strategies. Journal of Computational Science, 2013.

[40] N. Vijaykrishnan, M. Kandemir, S. Kim, S. Tomar, A. Sivasubramaniam, and M. J. Irwin. Energy behavior of java applications from the memory perspective. In JavaTM Virtual Machine Research and Technology Symposium, 2001.

[41] W. Yuan and K. Nahrstedt. Energy-efficient soft real-time cpu scheduling for mobile multimedia systems. In SOSP, 2003.

[42] Y. Zhang, G. Huang, X. Liu, W. Zhang, H. Mei, and S. Yang. Refactoring android java code for on-demand computation offloading. In OOPSLA, 2012. 